\title{
Digital Orthopantomography vs Cone Beam Computed Tomography—Part 2: A CBCT Analysis of Factors Influencing the Prevalence of Periapical Lesions
}

\author{
Elisa Bonfanti ${ }^{1}$, Marcello Maddalone ${ }^{2}$, Alberto Pellegatta ${ }^{3}$, Claudio Luigi Citterio ${ }^{4}$, Marco Baldoni ${ }^{5}$
}

\begin{abstract}
Aim: Cone beam computed tomography (CBCT) is the most refined and affordable method available today for the examination of an incoming patient for different dental pathologies. The aim of this paper is to evaluate the significance of some factors influencing the prevalence of apical periodontitis.

Materials and methods: An ortopantomography (OPT) and CBCT scan of the dental arches were examined for each of the selected 45 patients. The presence of apical periodontitis (AP) was compared for CBCT and OPT examination. Sensitivity, specificity, predictive values, and accuracy were calculated for CBCT, using OPT as a reference. The impact of protective/risk factors on the development of AP was examined.

Results: CBCT showed higher sensitivity (250\%), predictive values (111\%), accuracy (111\%), and specificity (101\%) than OPT. It was found to have higher sensitivity in all the dentition areas, especially where empty anatomical spaces or more radiotransparent structures have a strict relationship with the tooth apex and periapical structures like upper front area, premolar areas, and, especially, in the upper molar area. The prevalence of AP increased from 16 to $17 \%$ in the case of insufficient conservative restoration or $25 \%$ in the case of microleakage, $35-42 \%$ in the case of prosthetic restoration, $56-67 \%$ for posts, and 60 and $85 \%$, respectively, for inadequate endodontic treatment and missed canals.

Conclusion: $\mathrm{CBCT}$ plays a decisive role in the evaluation of molar areas and in the endodontic treatment planning, when a close relationship between the apex and important anatomical structures exists. Different risk factors with different relevance are identified.
\end{abstract}

Clinical significance: As CBCT-examined results show, coronal restorations are moderate-risk factors, while insufficient endodontic treatments and posts are high-risk factors for the development of AP.

Keywords: Apical lesion, CBCT, Cohort study, Digital orthopantomography, Radiographic assessment.

The Journal of Contemporary Dental Practice (2019): 10.5005/jp-journals-10024-2576

\section{INTRODUCTION}

Cone beam computed tomography (CBCT) is a method introduced approximately 15 years ago to visualize three-dimensional (3D) images of individual tooth or complete dentition, surrounding skeletal or periodontal tissues and to create 3D images of the area to be examined. ${ }^{1}$

CBCT can be a powerful tool in endodontic diagnosis, treatment planning, and follow-up, offering the opportunity to see the current pathosis affecting the apexes and the real state-of-the-art of their treatments, bypassing the disturbances created by surrounding tissues.

CT scans showed full reliability for the reproduction of the tooth inner anatomy and a technical evolution and refinement, the micro CT scan, is today currently used in endodontic research. ${ }^{2,3}$

In the following decades, the morphology of the canal system was studied using both the described techniques and other innovative ones, as well as in other disciplines. ${ }^{2-6}$

The knowledge of anatomy and conditions of the root canal system allows the clinician to better detail treatment alternatives or the presence and extension of an apical lesion. It could be useful in making the patient aware of his clinical status, predicting a prognosis, and supporting him in taking a more informed decision about his personalized treatment plan. ${ }^{7}$

Even if $\mathrm{CBCT}$ presents radiation doses that must be taken into consideration, when we select the diagnostic tool, clinical studies comparing the presence of periapical lesions in root-filled teeth with $\mathrm{CBCT}$ and periapical radiographs show that $\mathrm{CBCT}$ identifies at least $20 \%$ more lesions than periapical radiographs. ${ }^{7-11}$
${ }^{1-5}$ School of Dental Medicine, University of Milano-Bicocca, S. Gerardo Hospital, Monza, Italy

Corresponding Author: Marcello Maddalone, School of Dental Medicine, University of Milano-Bicocca, S. Gerardo Hospital, Monza, Italy, Phone: +39 3924655950, e-mail: marcello.maddalone@unimib.it

How to cite this article: Bonfanti E, Maddalone M, et al. Digital Orthopantomography vs Cone Beam Computed Tomography_Part 2: A CBCT Analysis of Factors Influencing the Prevalence of Periapical Lesions. J Contemp Dent Pract 2019;20(6):664-669.

Source of support: Nil

\section{Conflict of interest: None}

Since OPT fails to detect an important number of untreated radiolucencies, as demonstrated for periapical radiographs, its capacity in identifying the outcome of root canal therapies is questionable. Nevertheless, CBCT is currently not recommended, due to radiological considerations, for routine assessment of the outcome of root canal treatment, but its use is justified in research to provide a more objective indication of the outcomes. ${ }^{1}$

CBCT in fact produces images of the endodontic spaces, offering not only $3 \mathrm{D}$ assessment of the region of interest, but also generating images with a sufficient spatial resolution to allow detailed assessment of the tooth and the surrounding bone.

Moreover, if $\mathrm{CBCT}$ is not currently suggested as routine assessment to evaluate the outcome of root canal treatment, ${ }^{1}$ its use could surely be justified as a second-level examination to provide a more objective and sound examination.

(0) The Author(s). 2019 Open Access This article is distributed under the terms of the Creative Commons Attribution 4.0 International License (https://creativecommons. org/licenses/by-nc/4.0/), which permits unrestricted use, distribution, and non-commercial reproduction in any medium, provided you give appropriate credit to the original author(s) and the source, provide a link to the Creative Commons license, and indicate if changes were made. The Creative Commons Public Domain Dedication waiver (http://creativecommons.org/publicdomain/zero/1.0/) applies to the data made available in this article, unless otherwise stated. 
Root canal treatment and retreatment studies show variable success rates of $28-97.7 \%$ in different studies. ${ }^{12-15}$ Some studies have limitations like the use of inexperienced operators, ${ }^{16}$ others considered low recall rates. ${ }^{17,18}$

However, the fact that a single radiograph is two-dimensional is a major factor limiting its diagnostic ability. ${ }^{19}$ Other factors influencing the outcome in recognizing apical lesions are scanners themselves, the region of the jaw being scanned, superimposition or proximity of anatomical structures, exposure settings of the CBCT scanner, exposure time, size of the field of view (FOV), and the energy/potential $(\mathrm{kV}){ }^{20,21}$ The effective dose is also dependent on the region of the oral cavity being scanned. ${ }^{20-22}$

The aim of this research is to perform a retrospective standardized study to evaluate the significance of some factors influencing the prevalence of apical periodontitis using $C B C T$ in evaluating the presence of periapical lesions in a sample of 1,060 teeth.

\section{Materials and Methods}

Forty-five patients, 20 males and 25 females, aged between 19 years and 54 years, were randomly selected from our archive (University Milano Bicocca-Department of Dentistry, Monza, Italy) between 2013 and 2018. For each patient, a radiographic examination (CBCT) was evaluated.

From a theoretical pool of 1,440 teeth (from the 45 patients examined), only 1,060 teeth were present, while the other 380 teeth had previously been extracted or lost. One hundred and sixty-five teeth showed periapical lesions on CBCT. Patients with previous orthodontic treatment, impacted teeth, teeth with an immature apex, and residual roots were excluded from the sample.

CBCT scans were acquired with a Sirona Orthophos XG 3D hybrid imaging device (Sirona Dental), with an $8 \times 8 \mathrm{~cm}$ FOV, under the operating conditions of $85 \mathrm{kV}$ and $7 \mathrm{~mA}$. The high definition mode used for this study provided for a $360^{\circ}$ rotation of the X-ray source and a scan time of 14.4 seconds with a continuous exposure. This allowed acquiring 500 individual images and reducing the resolution from 160 to $100 \mu \mathrm{m}$, thus, obtaining detailed, highresolution volumes for the evaluation of the smallest structures. The X-ray dose for the $8 \times 8 \mathrm{~cm}$ FOV was 136 to $191 \mu \mathrm{Sv}$.

Risk factors that can cause apical periodontitis were analyzed

The prosthetic rehabilitation of the tooth has been analyzed in terms of the presence of posts and/or prosthetic crowns; the crowns have been classified into single crowns and bridges. The presence of caries, fillings, and endodontic treatment was noted. The quality of the canal treatment was evaluated by CBCT: in addition to identifying the presence of any untreated canals, the distance between the apical end of the root canal filling and the radiological apex was measured for each canal, and if this distance was more than $2 \mathrm{~mm}$, the filling was considered inadequate. Finally, the presence of the Schneiderian membrane and the presence of the inferior alveolar canal at less than $2 \mathrm{~mm}$ from the dental apices were annotated.
The results of the radiographic evaluation have been reported in frequency tables for the presence of apical lesions and for the other risk factors.

For each patient, an OPT made in the previous 2 months was also evaluated to compare detection rates.

\section{Results}

The prevalence of apical periodontitis was found to be $15.6 \%$, as 165 periapical lesions were identified on a total of 1,060 teeth included in the study.

CBCT identified two and a half times the number of apical lesions identified by OPT in the same group of patients (Table 1).

When the endo-conservative condition of each tooth was evaluated, it was observed that

- $37 \%$ of the endodontically treated teeth had apical lesions, while 63\% appeared healthy;

- if the length of the root canal filling was not adequate, $59 \%$ of the teeth had apical lesions, while the remaining $41 \%$ showed no signs of apical periodontitis;

- when an endodontically treated tooth had an untreated canal, teeth with apical lesions rose to $85 \%$, while the remaining $15 \%$ showed no sign of apical periodontitis;

- $17 \%$ of teeth with adequate coronal obturation had apical lesions, while $83 \%$ appeared healthy;

- $25 \%$ of teeth with caries or with an infiltrated reconstruction had signs of apical lesions, as opposed to the remaining $75 \%$.

When the prosthetic rehabilitation of the teeth was considered, it was observed that

- $42 \%$ of the crowned teeth had apical lesions, while $58 \%$ appeared healthy;

- bridge anchor crowns had a 30\% probability of having periapical lesion;

- if the prosthetically restored tooth also had a post, in $53 \%$ of cases, this showed apical lesion, while in $47 \%$, it appeared healthy (Fig. 1).

Of the 143 endodontically treated teeth, $94(66 \%)$ had a satisfactory canal obturation, while the remaining 49 (34\%) had inadequate apical extension filling. In the first group, 35 teeth had an apical lesion and 59 had healthy apices; in the second group, 29 presented an apical lesion and only 20 teeth had a healthy apex (Fig. 2).

Untreated canals were classified by the position and type. Twenty-eight untreated canals located in 20 teeth were identified, of which 2 (10\%) were upper first premolars, 4 (20\%) were upper or lower second molars, and 14 (70\%) were upper first molars (Fig. 3).

The most unidentified and consequently untreated canal of the first upper molar was found to be the second canal of the mesiobuccal root (MB2), with $50 \%$ of all untreated canals and $74 \%$ of the superior first molar untreated canals, while the distal (D) and mesiobuccal canal (MB1) were not treated in the remaining 21 and $5 \%$ of cases, respectively.

Table 1: Revealed lesions in different dental positions by OPT and CBCT and percentage of lesions identified by CBCT compared to OPT

\begin{tabular}{lccccccccc}
\hline & All & Superior arch & Front teeth & Premolars & Molars & Inferior arch & Front teeth & Premolars & Molars \\
\hline CBCT & 165 & 92 & 15 & 37 & 40 & 73 & 11 & 18 & 44 \\
OPT & 66 & 24 & 8 & 10 & 6 & 42 & 7 & 13 & 22 \\
Percentage & 250.0 & 383.3 & 187.5 & 370.0 & 666.7 & 173.8 & 157.1 & 138.5 & 200.0 \\
\hline
\end{tabular}




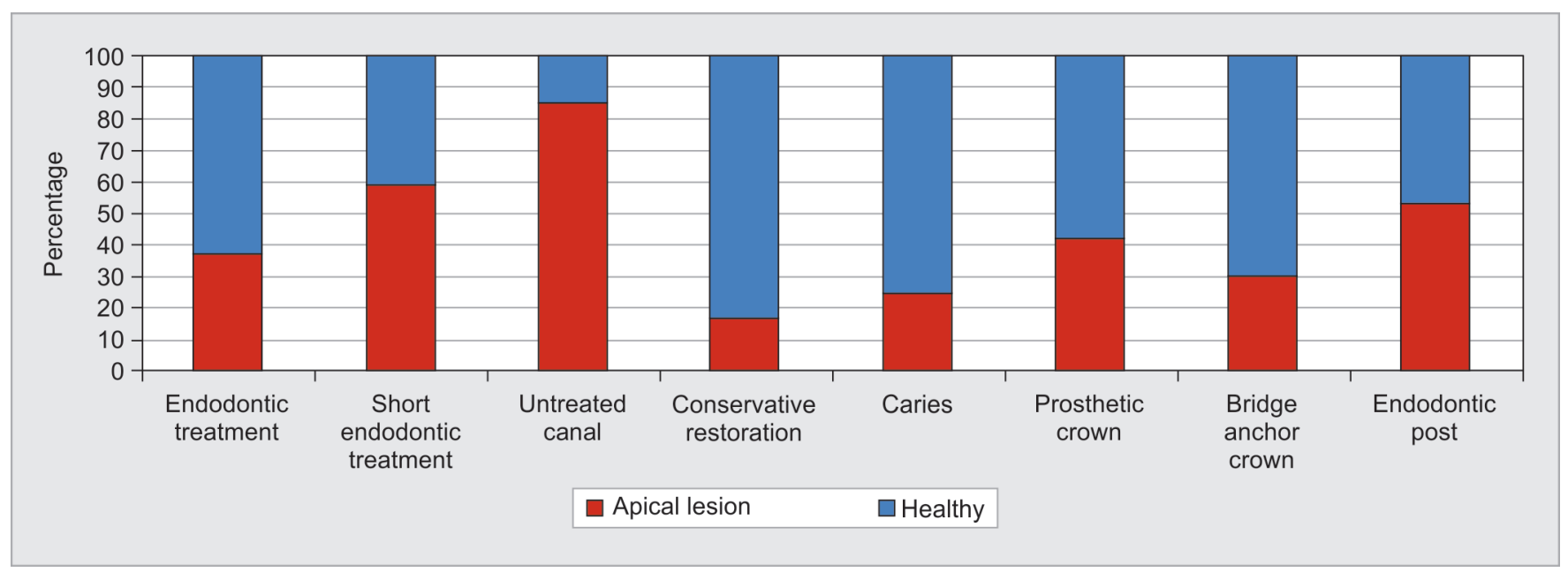

Fig. 1: Percentages of pathological and healthy apices in the different risk categories considered in this study

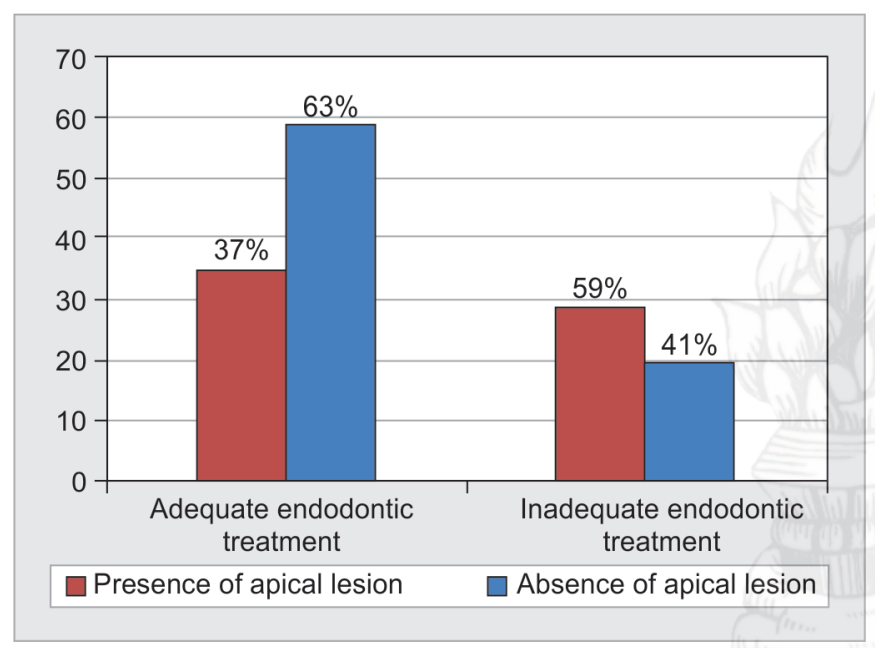

Fig. 2: Frequency of distribution of endodontic treatments evaluated in terms of root canal obturation length

The most frequently untreated canals in second superior molars were the mesiobuccal $(60 \%)$, whereas the distal canals were less frequently missed (40\%).

The mesiolingual canal was the only one that could be missed in the second inferior molar and similarly the palatal canal for the first superior premolar.

Subsequently, the relationship between the development of apical lesions and the presence of an endodontic treatment in teeth that had to be prosthetically rehabilitated was evaluated. Teeth presenting both apical and coronal seals had $48 \%$ probability of having an apical lesion. Lower percentages were obtained when the endodontically treated tooth was exposed in the oral cavity without any prosthetic restoration (39\%). On the contrary, if the prosthetically treated tooth had not undergone any endodontic treatment, the risk of incurring a periapical lesion was $26 \%$.

The ability of the two radiographic methods to intercept the carious processes was investigated, too. The presence of 143 caries detected by orthopantomography was confirmed when the CBCT was examined. However, 87 additional radiolucent areas were identified using CBCT and 27 were visible only on the OPT. Frequently, it was impossible to detect radiolucent lesions on the OPT because

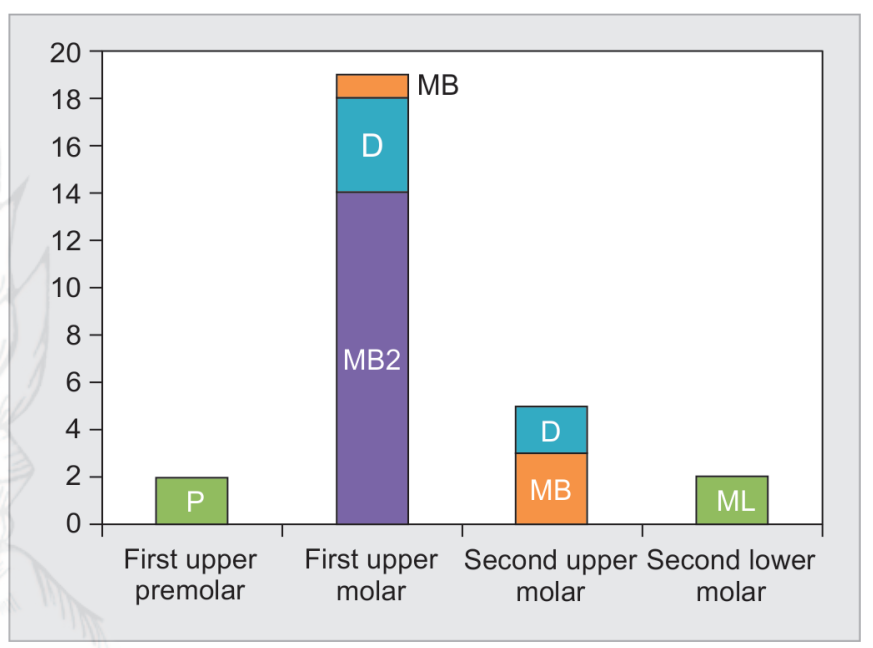

Fig. 3: Frequency of distribution of untreated canals divided by the type of tooth

of the superimposition of radiopaque structures, such as prosthetic crowns and adjacent teeth in the case of dental crowding.

\section{Discussion}

\section{Validity of the Diagnostic Test}

CBCT showed much higher sensitivity (250\%), higher predictive values and diagnostic accuracy (111\%), and similar specificity (101\%) compared to OPT for the diagnosis of apical lesions (Table 2).

\section{Endodontic Treatment Quality}

Outcome studies, ${ }^{23-25}$ agree that treatments in which the obturation material reaches $0-2 \mathrm{~mm}$ from the radiographic apex are associated with a greater likelihood of success compared to short treatments (more than $2 \mathrm{~mm}$ from the radiographic apex) or long ones (extruded material).

Table 2: Summary table of sensitivity, specificity, positive and negative predictive values, and diagnostic accuracy of CBCT when compared to those of digital orthopantomography

\begin{tabular}{lllll}
\hline Sensitivity & Specificity & Ppv & Npv & Accuracy \\
\hline $250 \%$ & $101 \%$ & $111 \%$ & $111 \%$ & $111 \%$ \\
\hline
\end{tabular}


Actually, we found that the probability to find signs of periapical lesions is $37 \%$ in the case of teeth with adequate treatment, while it reaches $59 \%$ for teeth in which the root canal filling does not present an adequate extension in a 3D perspective. The finding that the probability of complete resolution of the apical pathology is significantly higher for adequately extended treatments confirms the fact that the elimination of apical lesions strongly depends on the quality of instrumentation and root canal obturation procedure. ${ }^{24}$

The prevalence of untreated infected canals in endodontically treated teeth is $12 \%$, while Karabucak et al. ${ }^{26}$ found a higher value (23\%).

The tooth with the highest incidence of missed canals is the maxillary left first molar, with $50 \%$ of all untreated canals, followed by the right one, with $18 \%$. It seems that the endodontic treatment of the left superior side of dentition is easier for the right-handed operator and that the visibility is superior to that in the contralateral side. Lower or similar percentages were obtained for the superior first premolar (7\%) and for the superior (18\%) and inferior (7\%) second molars.

Missed canals have an $85 \%$ probability of showing signs of an apical lesion (Figs 4 and 5). The properly treated canal is devoid of pulp residues, it is dry and disinfected and it represents an unfavorable environment for bacterial survival. ${ }^{27}$

However, in cross-sectional studies based on retrospective radiographic information, it is not possible to discern a persistent lesion from a recent onset lesion or from an incomplete healing lesion (scar tissue). ${ }^{23,28}$ Therefore, the prevalence of apical lesions in endodontically treated teeth could be the result of an overestimation.

\section{Posts}

In our study, we observed that the presence of an endodontic post significantly increased the risk of developing a periapical lesion, regardless of the type of prosthetic restoration. This question has been rarely debated in the literature and previous findings are contradictory, but our results are clear: the probability of the presence of an apical lesion increases by $25 \%$ if a post is used for prosthetic rehabilitation. It seems that the post, although it has a modulus of elasticity similar to that of dentin, predisposes the tooth to micro-fractures and post-debonding due to the discharge of occlusal forces. ${ }^{29-31}$

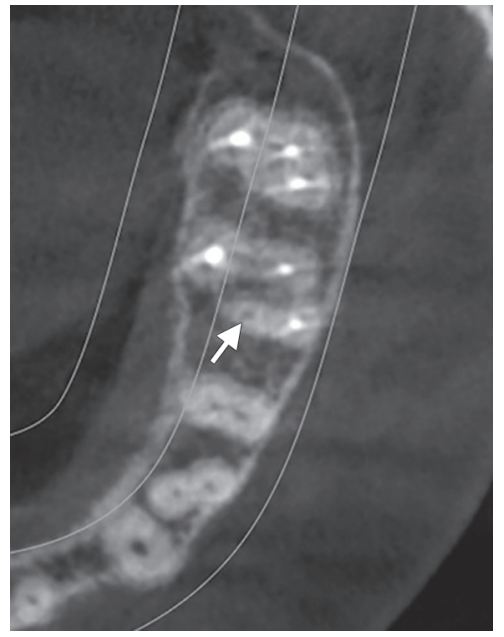

Fig. 4: Axial section of $C B C T$ showing the untreated $M B 2$ canal that was invisible on OPT

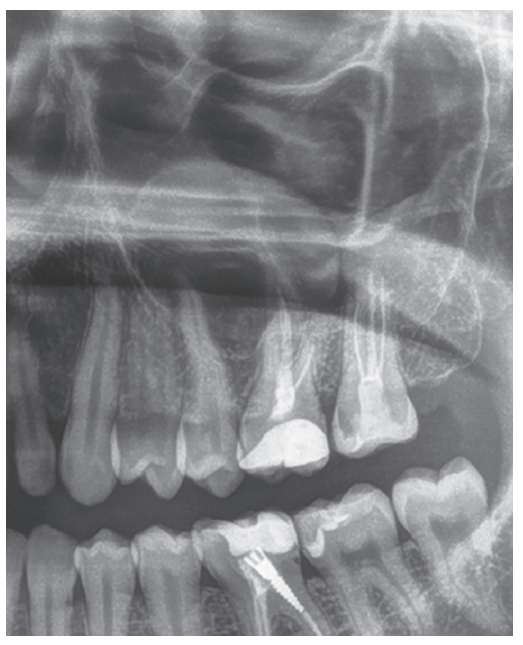

Fig. 5: The untreated MB2 canal on OPT

\section{Apical and Coronal Seal}

It was observed that teeth with both an endodontic treatment and a prosthetic restoration had a higher risk (48\%) of having apical lesions compared to the teeth with the endodontic seal alone and protected only by a composite restoration (39\%). Teeth that had been rehabilitated with a prosthetic restoration without root canal treatment had a further lower probability of presenting apical lesions (26\%): this is due to the initial unfavorable condition, rather than by an effective operative risk. In fact, only healthy teeth can be prosthetically rehabilitated without endodontic treatment, while endodontically treated teeth were teeth with a greater risk of developing periapical pathology or frankly pathological teeth from the beginning. Apical lesions in endodontically treated teeth could also be the result of the healing process.

The unexpected discrepancy between teeth with corono-apical seal and those with only endodontic seal is due to the presence of posts, in the first of these groups, which are risk factors for root fractures and bacterial infiltration.

\section{Coronal Restoration}

When the prevalence of apical lesions was investigated for prosthetically restored teeth, it was observed that the single crown is associated with a higher risk of lesion development (42\%) compared to the restorations involving adjacent teeth (30\%). It seems that the single prosthetic restoration predisposes the abutment to a greater risk of coronal infiltration, lack of coronal seal and, therefore, to a consequent risk of endodontic failure. Conversely, the increased extent and surface of multiple restorations is the determining factor in the neutralization of some of the mechanical forces that tend to dislocate the prosthetic reconstruction. Single crowns are more exposed to transverse or rotational forces, contrasted only by the presence of cement.

However, possible confounding factors have been analyzed. Some authors (Maddalone et al. ${ }^{32}$ and Ivanović et al. ${ }^{33}$ ) signaled posts as the predisposing factor to root fractures. Actually, posts in bridge crowns were found to be fewer than posts in single crowns (25 and $8 \%$, respectively), but, when we excluded the teeth with posts and considered only the teeth without posts, the discrepancy between single crowns and bridges did not change. The same result was obtained when the second possible confounding factor, i.e., the endodontic treatment quality, was 
examined. Therefore, since we did not find real confounding factors, the observed discrepancy between single crowns and bridges was connected to the superior exposure of single crowns to dislocating forces.

The literature contains very few studies ${ }^{34,35}$ which investigated the effects of caries and tooth partial reconstruction on periapical health.

In our study, teeth with adequate restorations had signs of apical periodontitis in $17 \%$ of cases, regardless of the material used, while infiltrated reconstructions and carious lesions were associated with apical lesions in $25 \%$ of cases. Therefore, the presence of an adequate restoration does not represent a high-risk factor for apical health, if the instrumentation and root filling procedures are correctly performed.

Although the beam-hardening artifacts due to the presence of radiopaque materials (enamel, filling materials, and prosthetic restorations) are limiting factors in the identification of caries, Orthophos XG 3D has already shown, in a previous study, ${ }^{36}$ a much higher sensitivity and specificity than other devices for this specific purpose.

\section{Conclusion}

CBCT shows a superior diagnostic accuracy in all the dental areas and it plays a strategic role in the diagnosis of apical lesions of the maxillary arch, especially in the molar area, where the minimum sensitivity was observed for OPT and in the lower molar area, where the accuracy is poor. $\mathrm{CBCT}$ also has an important role in the endodontic treatment planning, both surgical and nonsurgical, when the probability of incurring accessories canals or a complex root canal system is high. Moreover, it provides essential support in the identification of endodontic failures, like in the detection of previously missed canals.

Our study has also pointed out that the insufficient extension of root canal filling, the presence of missed canals, and posts are important risk factors for the development of apical pathologies, whereas the coronal restorations represent moderate risk factors (Fig. 1).

\section{Clinical Significance}

CBCT could be useful in identifying hidden periapical lesion and it is an important diagnostic tool, especially when posts, inadequate restorations, and caries are present or missed canals are suspected.

\section{References}

1. Patel $S$, Durack $C$, et al. Cone beam computed tomography in endodontics - a review. Int Endod J 2015;48(1):3-15. DOI: 10.1111/ iej.12270.

2. Citterio F, Pellegatta A, et al. Analysis of the apical constriction using micro-computed tomography and anatomical sections. In: Giornale Italiano Di Endodonzia, 2014; pp. 41-45, vol. 28. DOI: 10.1016/j. gien.2014.05.001.

3. Venino PM, Citterio $\mathrm{CL}$, et al. A micro-computed tomography evaluation of the shaping ability of two nickel-titanium instruments, HyFlex EDM and ProTaper next. J Endod 2017;43(4):628-632. DOI: 10.1016/j.joen.2016.11.022.

4. Maddalone $M$, Ferrari $M$, et al. Use of miniscrew implants in orthodontic distal movement [Utilizzo delle miniviti nelle meccaniche ortodontiche di distalizzazione]. Dent Cadmos 2010;78(8):97-105.

5. Maddalone $M$, Ferrari $M$, et al. Intrusive mechanics in orthodontics with the use of TAD's [Utilizzo delle miniviti nelle meccaniche ortodontiche di intrusione]. Dent Cadmos 2010;78(7):97-106.
6. Maddalone M. The association between the psychological status and the severity of facial deformity in orthognathic patients. Angle Orthod 2012;82(3):396-402. DOI: 10.2319/060211-363.

7. Patel $\mathrm{S}$, Wilson $\mathrm{R}$, et al. The detection of periapical pathosis using digital periapical radiography and cone beam computed tomography part 2: a 1 year post-treatment follow-up. Int Endod J 2012;45(8): 711-723. DOI: 10.1111/j.1365-2591.2012.02076.x.

8. Lofthag-Hansen S, Huumonen S, et al. Limited cone-beam CT and intraoral radiography for the diagnosis of periapical pathology. Oral Surg Oral Med Oral Pathol Oral Radiol Endod 2007;103(1):114-119. DOI: 10.1016/j.tripleo.2006.01.001.

9. Estrela C, Bueno MR, et al. Accuracy of cone beam computed tomography and panoramic and periapical radiography for detection of apical periodontitis. J Endod 2008;34(3):273-279. DOI: 10.1016/j. joen.2007.11.023.

10. Low KMT, Dula K, et al. Comparison of periapical radiography and limited cone-beam tomography in posterior maxillary teeth referred for apical surgery. J Endod 2008;34(5):557-562. DOI: 10.1016/ j.joen.2008.02.022.

11. Friedman S. Prognosis of initial endodontic therapy. Endod Top 2002;2(1):59-88. DOI: 10.1034/j.1601-1546.2002.20105.x.

12. Davies A, Mannocci F, et al. The detection of periapical pathoses in root filled teeth using single and parallax periapical radiographs versus cone beam computed tomography - a clinical study. Int Endod J 2015;48(6):582-592. DOI: 10.1111/iej.12352.

13. Theodorakou C, Walker A, et al. Estimation of paediatric organ and effective doses from dental cone beam CT using anthropomorphic phantoms. Br J Radiol 2012;85(1010):153-160. DOl: 10.1259/bjr/19389412.

14. Danin J, Strömberg T, et al. Clinical management of nonhealing periradicular pathosis. Surgery versus endodontic retreatment. Oral Surg Oral Med Oral Pathol Oral Radiol Endod 1996;82(2):213-217. DOI: 10.1016/S1079-2104(96)80259-9.

15. Gorni FGM, Gagliani MM. The outcome of endodontic retreatment: a 2 year follow-up. J Endod 2004;30(1):1-4. DOI: 10.1097/00004770200401000-00001.

16. de Chevigny C, Dao TT, et al. Treatment outcome in endodontics: the Toronto study-phase 4: initial treatment. J Endod 2008;34(3):258-263. DOI: 10.1016/j.joen.2007.10.017.

17. Sundqvist G, Figdor D, et al. Microbiologic analysis of teeth with failed endodontic treatment and the outcome of conservative re-treatment. Oral Surg Oral Med Oral Pathol Oral Radiol Endod 1998;85(1):86-93. DOI: 10.1016/S1079-2104(98)90404-8.

18. Farzaneh M, Abitbol S, et al. Treatment outcome in endodontics: the Toronto study. Phases I and II: orthograde retreatment. J Endod 2004;30(9):627-633. DOI: 10.1097/01.DON.0000129958.12388.82.

19. Patel S, Dawood A, et al. Detection of periapical bone defects in human jaws using cone beam computed tomography and intraoral radiography. Int Endod J 2009;42(6):507-515. DOI: 10.1111/j.13652591.2008.01538.x.

20. Huumonen $S, \varnothing$ rstavik D. Radiological aspects of apical periodontitis. Endod Top 2002;1:3-25. DOI: 10.1034/j.1601-1546.2002.10102.x.

21. Pauwels $\mathrm{R}$, Beinsberger J, et al. Effective dose range for dental cone beam computed tomography scanners. Eur J Radiol 2012;81(2): 267-271. DOI: 10.1016/j.ejrad.2010.11.028.

22. Ivanović $\mathrm{M}$, Jovičić $\mathrm{O}$, et al. Oral manifestations of acute leukaemia. Srp Arh Celok Lek 2011;139(1-2):103-106.

23. Cakici E, Yildirim E, et al. Assessment of periapical health, quality of root canal filling, and coronal restoration by using cone-beam computed tomography. Niger J Clin Pract 2016;19(5):673. DOI: 10.4103/1119-3077.188697.

24. Liang YH, Li G, et al. The association between complete absence of post-treatment periapical lesion and quality of root canal filling. Clin Oral Investig 2012;16(6):1619-1626. DOI: 10.1007/s00784-011-0671-3.

25. Ng YL, Mann V, et al. Outcome of primary root canal treatment: systematic review of the literature - part 2. Influence of clinical factors. Int Endod J 2008;41(1):6-31. DOI: 10.1111/j.1365-2591.2007.01323.x.

26. Karabucak B, Bunes A, et al. Prevalence of apical periodontitis in endodontically treated premolars and molars with untreated canal: 
a cone-beam computed tomography study. J Endod 2016;42(4): 538-541. DOI: 10.1016/j.joen.2015.12.026.

27. Del Fabbro M. Le infezioni endodontiche. G Ital Endod 2009;23(1): 34-37.

28. Gambarini G, Piasecki L, et al. Cone-beam computed tomography in the assessment of periapical lesions in endodontically treated teeth. Eur J Dent 2018;12(1):136-143. DOI: 10.4103/ejd.ejd_320_17.

29. Sorrentino R, Di Mauro MI, et al. Complications of endodontically treated teeth restored with fiber posts and single crowns or fixed dental prostheses - a systematic review. Clin Oral Investig 2016;20(7):1449-1457. DOI: 10.1007/s00784-016-1919-8.

30. Signore A, Benedicenti S, et al. Long-term survival of endodontically treated, maxillary anterior teeth restored with either tapered or parallel-sided glass-fiber posts and full-ceramic crown coverage. J Dent 2009;37(2):115-121. DOI: 10.1016/j.jdent.2008.10.007.

31. Farah RI, Aldakhili AS, et al. A radiographic study of the association between apical periodontitis and technical quality of intraradicular posts and root canal fillings: a cross-sectional study in Qassim Region,
Saudi Arabia. Contemp Clin Dent 2017;8(4):538-544. DOI: 10.4103/ ccd.ccd.

32. Maddalone M, Gagliani M, et al. Prevalence of vertical root fractures in teeth planned for apical surgery. A retrospective cohort study. Int Endod J 2018;51(9):969-974. DOI: 10.1111/iej.12910.

33. Ivanović $\mathrm{M}$, Jovičić $\mathrm{O}$, et al. Prevention of oral diseases in children with acute leukaemia. Srp Arh Celok Lek 2011;139(3-4):242-247.

34. Ricucci D, Gröndahl K, et al. Periapical status of root-filled teeth exposed to the oral environment by loss of restoration or caries. Oral Surg Oral Med Oral Pathol Oral Radiol Endod 2000;90(3):354-359. DOI: $10.1067 /$ moe. 2000.108802 .

35. Dawson VS, Petersson K, et al. Periapical status of root-filled teeth restored with composite, amalgam, or full crown restorations: a cross-sectional study of a Swedish adult population. J Endod 2016;42(9):1326-1333. DOI: 10.1016/j.joen.2016.06.008.

36. Gaalaas $L$, Tyndall D, et al. Ex vivo evaluation of new 2D and 3D dental radiographic technology for detecting caries. Dentomaxillofacial Radiol 2016;45(3):1-10. DOI: 10.1259/dmfr.20150281. 